\title{
Task frequency influences stimulus-driven effects on task selection during voluntary task switching
}

\author{
Catherine M. Arrington • Kaitlin M. Reiman
}

Published online: 24 June 2015

(C) Psychonomic Society, Inc. 2015

\begin{abstract}
Task selection during voluntary task switching involves both top-down (goal-directed) and bottom-up (stimulus-driven) mechanisms. The factors that shift the balance between these two mechanisms are not well characterized. In the present research, we studied the role that task frequency plays in determining the extent of stimulus-driven task selection. In two experiments, we used the basic paradigm adapted from Arrington (Memory \& Cognition, 38, 991-997, 2008), in which the effect of stimulus availability serves as a marker of stimulus-driven task selection. A number and letter appeared on each trial with varying stimulus onset asynchronies, and participants performed either a consonant/vowel or an even/odd judgment. In Experiment 1, participants were instructed as to the relative frequency with which each task was to be performed (i.e., 50/50, 60/40, or $75 / 25$ ) and were further instructed to make their transitions between tasks unpredictable. In Experiment 2, participants were given no instructions about how to select tasks, resulting in naturally occurring variation in task frequency. With both instructed (Exp. 1) and naturally occurring (Exp. 2) relative task frequencies, the less frequently performed task showed a greater effect of stimulus availability on task selection, suggestive of a larger influence of stimulusdriven mechanisms during task performance for the less frequent task. When goal-directed mechanisms of task choice are engaged less frequently, the relative influence of the stimulus environment increases.
\end{abstract}

Electronic supplementary material The online version of this article (doi:10.3758/s13423-014-0777-0) contains supplementary material, which is available to authorized users.

C. M. Arrington $(\bowtie) \cdot$ K. M. Reiman

Department of Psychology, Lehigh University, 17 Memorial Drive

E., Bethlehem, PA 18015, USA

e-mail: kate.arrington@lehigh.edu
Keywords Task switching $\cdot$ Executive control $\cdot$ Cognitive control $\cdot$ Automaticity $\cdot$ Attentional control

In multitask environments, task selection results from the interplay of goal-directed intentions and environmental influences. The balance between top-down and bottom-up mechanisms of behavioral control is of interest to researchers studying both mental and neural processing (Haggard, 2008; Krieghoff, Waszak, Prinz, \& Brass, 2011). In the present research, we considered how selection mechanisms are influenced by the relative frequency of tasks and what this effect of frequency says about the cognitive control mechanisms involved in task selection.

Voluntary task switching (VTS) was developed to study cognitive control in multitask environments (Arrington \& Logan, 2004). VTS requires participants to choose which task to perform on a series of bivalent stimuli, with only general instructions on how to select tasks (e.g., in a random sequence) rather than explicit cues dictating the appropriate task. Task performance during VTS is similar to that derived from other task-switching paradigms, showing robust switch costs that decrease as the time between trials increases (Arrington \& Logan, 2005; Demanet \& Liefooghe, 2014). VTS also provides dependent measures of task selection processes, for both specific tasks and task transitions. Past studies have shown that task selection is influenced by various top-down factors, consistent with the interpretation that VTS captures aspects of intentional control. Task switching decreases with decreased preparation time (Arrington \& Logan, 2005), increased working memory load (Demanet, Verbruggen, Liefooghe, \& Vandierendonck, 2010), and greater response conflict (Orr, Carp, \& Weissman, 2012).

The environment also influences task selection during VTS. Stimulus repetition increases task repetition (Mayr \& Bell, 2006), suggesting that stimuli can prime task selection. 
Participants are more likely to choose to perform the task that was first performed on the initial exposure to a stimulus (Arrington, Weaver, \& Pauker, 2010). For displays containing multiple stimuli, both the timing (Arrington, 2008) and location of stimulus onset (Arrington \& Rhodes, 2010; Arrington $\&$ Weaver, in press) affect task selection. The strength of these bottom-up effects varies across individuals (Butler, Arrington, \& Weywadt, 2011) and situations (Demanet et al., 2010). Although this brief overview of VTS research suggests that task selection combines goal-directed and stimulus-driven factors, the balance between these mechanisms is poorly understood (Orr \& Weissman, 2011).

Accounts of the selection processes during VTS incorporate both top-down and bottom-up factors. Arrington and Logan (2005) proposed that competing heuristics that combine these two factors may guide task choice. The representativeness heuristic involves comparing a mental representation of a random sequence to the sequence of recently performed tasks held in working memory, to decide what next task would make this sequence most representative of randomness. This heuristic requires the active manipulation of information in working memory. The availability heuristic guides task selection on the basis of the most active task set. Task availability is determined by factors that are both topdown and bottom-up. Deviations from randomness, such as the repetition bias, result when the passive availability heuristic overcomes the more controlled use of the representativeness heuristic. Vandierendonck, Demanet, Liefooghe, and Verbruggen (2012) provided a more formal account of task selection in their chain-retrieval model, while still incorporating factors representing top-down and bottom-up processes. Individuals retrieve chains of tasks from long-term memory on the basis of a mental representation of randomness. Again, the actual performance of task sequences can deviate from randomness as a result of chain selection that is biased by either the ease of performance or stimulus-based priming.

In the present research, we explored the modulation of stimulus-based influences on task selection as a function of differential task frequency. The stimulus-based effect considered is the influence of stimulus availability, in which stimulus availability is operationalized as the stimulus onset asynchrony (SOA) between two stimuli affording different tasks (Arrington, 2008). When presented with two stimuli and instructed to perform the tasks randomly, participants select the task associated with the first stimulus with a probability, or $p(\mathrm{~S} 1)$, greater than chance. This effect decreases as the time between trials increases, which Arrington attributed to participants being more likely to have established an intention to perform a particular task following longer preparation times. If a top-down goal has not been formed during a short response-to-stimulus interval (RSI), then stimulus availability may influence task selection in a bottom-up fashion; however, if enough time has intervened between trials for a top-down goal to be established, then participants will search for the stimulus affording that task, regardless of the relative stimulus availability. Arrington framed this conclusion within Logan and Gordon's (2001) executive control theory of visual attention (ECTVA), which allows for the control of task performance based on a combination of parameters transferred topdown, from working memory to subordinate systems, and parameters determined bottom-up, by the stimulus environment. The bias parameter, $\beta$, represents the top-down bias toward responses associated with a particular task, whereas the evidence parameter, $\eta$, represents the bottom-up evidence for particular responses associated with the available stimuli. Thus, for long RSIs, the $\beta$ values are more likely to be set for a particular task prior to stimulus onset, and they dampen the effect of stimulus availability by overcoming the benefit gained from the earlier accumulation of evidence from S1. In the present study, we consider whether the relative frequency with which top-down goals are instantiated can modulate the bottom-up effect of stimulus availability. If relative task frequency is conceptualized as varying the likelihood that participants are prepared to perform a given task (i.e., $\beta$ values are set high for responses for that task), then task frequency is likely to influence the stimulus availability effect.

The experimental paradigms, including VTS, used to examine cognitive control are artificial in many ways. Of importance here, real-world tasks rarely occur equally often (e.g., one does not stir the noodles as frequently as the sauce when preparing pasta). When the top-down goals for different tasks are instantiated with differential frequencies, the influence of the bottom-up environmental factors on task selection may also vary. No research has looked directly at how the processes of task selection are influenced by relative task frequency; however, two related lines of task-switching research should be considered. First, the influence of transition frequency (i.e., the proportion of repetition vs. switch trials) has a substantial influence on switch costs in explicit task-cuing paradigms (Dreisbach \& Haider, 2006; Schneider \& Logan, 2006), which may suggest dynamic adjustment of control in varying switch contexts, but does not speak directly to task frequency. Second, relative task strength influences performance in task switching (Monsell, Yeung, \& Azuma, 2000). Asymmetric switch costs, with larger costs being associated with switching to the stronger task, occur in tasks that vary in preexperimental strength and intra-experimental practice (Yeung \& Monsell, 2003). In VTS, the difference in task strengths influences task selection processes, with participants performing the weaker task more often, presumably to avoid the difficult switch to the stronger task (Liefooghe, Demanet, \& Vandierendonck, 2010; Yeung, 2010). Drawing from this related literature and from the interaction between stimulus availability and preparation time found in Arrington (2008), we hypothesized that the less frequent task would show a larger influence of the external environment. This hypothesis was further supported by model 
predictions within ECTVA (Logan \& Gordon, 2001). Variations in the frequency with which a task is selected may influence the $\beta$ values associated with each task, in terms of both the likelihood and the strength with which these values are set in working memory and transmitted to the subordinate systems. The multiplicative relationship between $\beta$ and $\eta$ values results in a relative decrease in the effect of stimulus availability, captured in the $\eta$ values, when top-down goals, captured in the $\beta$ values, are greater.

\section{Experiment 1}

\section{Method}

Participants Sixteen individuals participated in exchange for course credit. All participants reported normal or corrected-tonormal vision.

Tasks and design The tasks were even/odd and consonant/ vowel judgments. The first independent variable was SOA, with eight levels: $0,17,66,100,133,167,200$, and $233 \mathrm{~ms}$. SOA varied randomly within each block. The second independent variable was task frequency, with three levels: 50/50, $60 / 40$, and $75 / 25$. Frequency varied across sessions. All participants completed the 50/50 condition in the first session, and the order of the other conditions was counterbalanced across participants in the remaining two sessions. The third independent variable was task bias, with two levels: high and low frequency. The tasks mapping to these values varied across participants.

Apparatus and stimuli All experimental procedures were programmed using E-Prime software (Psychology Software Tools Inc., Sharpsburg, PA) on Dell Dimension computers with 17-in. CRT monitors. Stimuli were presented in black 18point Courier New font on light gray backgrounds. The fixation screen contained a central cross, as well as pound sign (\#) placeholders at four possible target locations, positioned in a square around fixation. The leading edge of each placeholder was $1.25 \mathrm{~cm}$ from fixation. The target stimuli were the numbers $2-9$ and the letters A, B, C, E, I, L, U, and W. Responses were made on QWERTY keyboards using the "d," "f," " $\mathrm{j}$," and " $k$ " keys, with a counterbalanced mappings of one task per hand.

Procedure Trials began with the fixation screen for $500 \mathrm{~ms}$. A number and letter replaced the placeholders in two possible target locations with a variable SOA. The identity, position, and order of the two stimuli were selected randomly, with the constraint that $\mathrm{S} 1$ and $\mathrm{S} 2$ were equivalent across tasks. Both stimuli and the remaining placeholders were present until a response occurred.
Each session began with the instructions and 16 practice trials for each task. Participants then received the VTS instructions, informing them that both a letter and a number would appear on each trial, and that they should select one task to perform. Participants were further instructed regarding the frequency with which each task was to be performed, and finally were instructed to choose the tasks in a random order. (The exact instructions are provided in the Supplemental materials.) Participants completed a 16-trial VTS practice block, then completed eighteen 64-trial blocks. Participants received feedback after each block, showing their overall accuracy and the percentage of time on which the more frequent task was performed. Participants completed each frequency condition in separate 1-h sessions on different days within one week.

\section{Results and discussion}

Task choice was coded on the basis of the hand used to respond. The data were initially trimmed to remove the first trial of each block, error trials, and trials following errors, resulting in the removal of $11.6 \%$ of the trials. Overall, participants complied well with the frequency manipulation $(M \mathrm{~s}=50.6,58.4$, and 73.2 for the more frequent task in the 50/ $50,60 / 40$, and $75 / 25$ conditions, respectively), although they were slightly less biased than instructed in both the 60/40, $t(15)=2.7, p<.05$, and 75/25, $t(15)=4.2, p<.05$, conditions. Importantly, task selection varied significantly as a function of instructions, $F(2,30)=692.6, p<.05, \eta_{\mathrm{p}}{ }^{2}=.979$, with LSD contrasts showing highly significant differences among all of the conditions. Participants' choice data showed the standard repetition bias, with the probabilities of switching at .304, .290 , and .257 for the $50 / 50,60 / 40$, and $75 / 25$ conditions, respectively, but this bias did not differ significantly as a function of frequency condition, $F(2,30)=2.4, p=.11, \eta_{\mathrm{p}}{ }^{2}$ $=.136$. Reaction time (RT) analyses were not central to the research question, but appear in the Supplemental materials.

The effect of stimulus availability was captured in the probability of performing the task associated with the first stimulus to appear. These $p(\mathrm{~S} 1)$ values appear in Table 1, and were analyzed in an 8 (SOA: $0,17,66,100,133,167,200$, or $233 \mathrm{~ms}) \times 2$ (Task Bias: less frequent or more frequent) $\times 3$ (Frequency: 50/50, 60/40, or 75/25) repeated measures analysis of variance (ANOVA). We found a significant effect of SOA, $F(7,105)=4.5, p<.05, \eta_{\mathrm{p}}{ }^{2}=.229$, with $p(\mathrm{~S} 1)$ values generally increasing across SOAs monotonically until $167 \mathrm{~ms}$, where they appeared to reach asymptote. There was a marginally significant effect of task bias, $F(1,15)=4.0, p=.064, \eta_{\mathrm{p}}{ }^{2}$ $=.211$, with $p(\mathrm{~S} 1)$ values being larger for the less frequent $(M$ $=.552)$ than for the more frequent $(M=.540)$ task. Additionally, the interaction of task bias and frequency was also marginally significant, $F(2,30)=3.1, p=.058, \eta_{\mathrm{p}}{ }^{2}=$ .173 . This analysis considered all of the frequency conditions, 
Table 1 Means and standard errors of the probability of selecting the task associated with the first stimulus to appear, as a function of frequency condition (Exp. 1 only), task bias, and SOA for Experiments 1 and 2

\begin{tabular}{|c|c|c|c|c|c|c|c|c|c|c|}
\hline \multirow{2}{*}{$\begin{array}{l}\text { Frequency } \\
\text { condition }\end{array}$} & \multirow[t]{2}{*}{ Task bias } & & \multicolumn{8}{|c|}{ SOA } \\
\hline & & & 0 & 17 & 66 & 100 & 133 & 167 & 200 & 233 \\
\hline \multicolumn{11}{|c|}{ Experiment 1} \\
\hline \multirow[t]{4}{*}{$50 / 50$} & \multirow[t]{2}{*}{ Less frequent } & Mean & .495 & .510 & .540 & .547 & .557 & .560 & .577 & .585 \\
\hline & & $S E$ & .011 & .014 & .019 & .025 & .020 & .024 & .028 & .025 \\
\hline & \multirow{2}{*}{$\begin{array}{l}\text { More } \\
\text { frequent }\end{array}$} & Mean & .492 & .504 & .537 & .561 & .560 & .565 & .562 & .574 \\
\hline & & $S E$ & .013 & .013 & .018 & .022 & .020 & .021 & .021 & .022 \\
\hline \multirow[t]{4}{*}{$60 / 40$} & \multirow[t]{2}{*}{ Less frequent } & Mean & .509 & .544 & .561 & .543 & .561 & .594 & .581 & .589 \\
\hline & & $S E$ & .014 & .019 & .024 & .030 & .028 & .035 & .033 & .033 \\
\hline & \multirow{2}{*}{$\begin{array}{l}\text { More } \\
\text { frequent }\end{array}$} & Mean & .509 & .526 & .544 & .539 & .553 & .576 & .567 & .581 \\
\hline & & $S E$ & .009 & .014 & .019 & .025 & .026 & .025 & .027 & .029 \\
\hline \multirow[t]{4}{*}{$75 / 25$} & \multirow[t]{2}{*}{ Less frequent } & Mean & .525 & .513 & .549 & .544 & .588 & .581 & .551 & .540 \\
\hline & & $S E$ & .022 & .026 & .023 & .027 & .037 & .030 & .028 & .033 \\
\hline & \multirow{2}{*}{$\begin{array}{l}\text { More } \\
\text { frequent }\end{array}$} & Mean & .511 & .504 & .528 & .530 & .532 & .534 & .534 & .525 \\
\hline & & $S E$ & .008 & .009 & .012 & .010 & .012 & .014 & .014 & .014 \\
\hline \multicolumn{11}{|c|}{ Experiment 2} \\
\hline \multirow[t]{2}{*}{-} & \multirow[t]{2}{*}{ Less frequent } & Mean & .507 & .540 & .618 & .580 & .631 & .648 & .695 & .697 \\
\hline & & $S E$ & .008 & .021 & .027 & .033 & .039 & .034 & .038 & .045 \\
\hline \multirow[t]{2}{*}{-} & \multirow{2}{*}{$\begin{array}{l}\text { More } \\
\text { frequent }\end{array}$} & Mean & .493 & .529 & .596 & .556 & .601 & .610 & .636 & .628 \\
\hline & & $S E$ & .006 & .009 & .025 & .032 & .031 & .030 & .031 & .038 \\
\hline
\end{tabular}

including the 50/50 condition. Because the 50/50 condition was completed first, participants were unaware that the tasks would differ in frequency in the later sessions, and the assignment of tasks to task bias conditions was not meaningful because both tasks occurred equally often. An analysis including only the conditions in which the two tasks differed in frequency showed that the effect of task bias on $p(\mathrm{~S} 1)$ values was significant, $F(1,15)=5.0, p<.05, \eta_{\mathrm{p}}{ }^{2}=.249$, but did not differ as a function of frequency, $F(1,15)=1.6, p=.23$, or SOA, $F(7,105)=1.3, p=.27$.

The effect of stimulus availability biased participants to select the task associated with the first stimulus to appear, demonstrating a bottom-up influence on task selection (Arrington, 2008). As we hypothesized, the bias was stronger for the less frequent task in both the $60 / 40$ and $75 / 25$ conditions. Thus, the strength of the bottom-up effect appears to be modulated by the frequencies with which participants are performing the two tasks.

\section{Experiment 2}

In the real world, factors such as relative importance, difficulty, and personal preference combine to determine task frequency, rather than instructions. In Experiment 2, we considered whether such naturally occurring biases in task frequency affect the influence of external stimuli on task choice. In the absence of instructions regarding how to select and sequence tasks, participants performing VTS often select one task with greater frequency (Arrington, Reiman, \& Weaver, 2014; Kessler, Shencar, \& Meiran, 2009). With tasks, stimuli, and timing that were identical to those elements of Experiment 1, participants performed VTS without instructions on how to select the tasks. A range of frequencies in task bias arose, and we examined the effect of stimulus availability for the more and less frequent tasks on the basis of participants' choices.

Method

Participants Sixty-six individuals participated for course credit. All participants reported normal or corrected-tonormal vision.

Procedure The apparatus, stimuli, tasks, and trial procedure were identical to those aspects of Experiment 1. The instructions regarding task selection differed. After singletask practice, participants were instructed that both a number and a letter would appear on each trial, and that they must pick which task to perform. However, they were given no specific instructions as to the frequency or order with which the tasks should be selected (see the Supplemental materials). Participants then completed one VTS practice block and eighteen 64-trial data collection blocks in a single 1-h session. 


\section{Results and discussion}

The lack of experimental control resulting from the free nature of the instructions to participants resulted in the need to exclude a large number of participants who provided no useable data. We removed 30 participants who performed the less frequent task on less than $5 \%$ of trials $(M=0.1 \%)$. Of the remaining participants, we removed 20 who switched tasks on less than $5 \%$ of trials $(M=1.0 \%)$, leaving 16 participants. These data were trimmed as in Experiment 1, resulting in the removal of $12.4 \%$ of the trials.

On average, participants selected their preferred task on $62.3 \%(S D=11.6 \%)$ of trials, representing a significant bias toward the preferred task, $t(15)=4.2, p<.05$. RT analyses (see the Supplemental materials) indicated that participants were also faster to respond to their preferred task. Participants also showed the expected repetition bias, with an average $p$ (switch) of .199, which differed significantly from chance, $t(15)=8.7, p<.05$. The $p(\mathrm{~S} 1)$ values (Table 1$)$ replicated the pattern found in Experiment 1, when task bias was manipulated. An 8 (SOA: 0, 17, 66, 100, 133, 167, 200, or $233 \mathrm{~ms}$ ) $\times 2$ (Task Bias: less frequent or more frequent) repeated measures ANOVA was performed. The $p(\mathrm{~s} 1)$ values generally increased across SOAs, though in a nonmonotonic fashion, $F(7,105)=$ $11.4, p<.05, \eta_{\mathrm{p}}{ }^{2}=.432$. Importantly, the $p(\mathrm{~S} 1)$ values were significantly larger for the less frequent $(M=.614)$ than for the more frequent $(M=.581)$ task, $F(1,15)=12.8, p<.05, \eta_{\mathrm{p}}{ }^{2}=$ .460. Task bias and SOA did not interact, $F(7,105)=1.3, p=$ .237 .

The bottom-up effect of stimulus availability was modulated by variations in task frequency, which in this case were naturally occurring. The less frequent task showed larger effects of the external environment on task selection. Although this basic effect of task bias replicated our previous results, it is worth noting that the $p(\mathrm{~S} 1)$ magnitude was overall larger in Experiment 2 than in any condition in Experiment 1 (see the Supplemental materials for cross-experiment analyses). This difference between the experiments likely indicates a smaller influence of bottom-up stimulus effects on task selection in Experiment 1, in which the more constrained instructions regarding task frequency and order may have resulted in stronger top-down goals.

Some consideration should be given to the exclusion of a large number of participants from this study. Only a quarter of the participants run in Experiment 2 provided useable data, because most participants either exclusively performed one task or switched between tasks at a very low rate. Although this pattern is consistent with other studies of VTS using unconstrained instructions (Arrington et al., 2014), it appears to contrast with studies of free-choice decisions that allow participants to freely decide between two tasks or responses (Soon, He, Bode, \& Haynes, 2013), in which choice does not show a strong repetition bias, even without explicit instructions to make the choices at random. Critical factors such as the type of task and the speed at which the decision must be performed differ between these paradigms, and our understanding of free-choice processes would benefit from a systematic investigation of the differences between these situations.

\section{General discussion}

In the present study, we addressed the question of whether the bottom-up influences on task selection vary as a function of relative task frequency. Across two different procedures, variations in task frequency resulted in similar shifts in the influence of the external environment on task selection. Both when task choice was instructed (Exp. 1) and when it was naturally occurring (Exp. 2), the less frequently performed task showed a greater effect of stimulus availability on task selection. Specifically, whereas the probability of performing the task associated with the first stimulus to appear increased with increasing SOA for both tasks, the $p(\mathrm{~S} 1)$ values were significantly larger for the less frequent task.

The larger effects of stimulus availability on task choice for the less frequent task fit within the competing-heuristics (Arrington \& Logan, 2005) and chain-retrieval (Vandierendonck et al., 2012) accounts of task choice. Although both accounts were developed to capture choice processes under random instructions, the basic approach of selecting tasks to conform to a mental representation of a representative task sequence (or to all possible task sequences in long-term memory, in the case of the chain-retrieval model) can easily accommodate tasks of varying frequencies. When one is performing one task more frequently, either by instruction or by preference, the mental representation will favor the more frequent task. Thus, on any given trial the more frequent task is more likely to be selected intentionally on the basis of top-down control, or in Logan and Gordon's (2001) ECTVA terminology, the $\beta$ values for the more frequent task are more likely to be transmitted to the subordinate systems. However, the influence of bottom-up processes associated with stimulus availability, an advantage resulting from greater environmental evidence ( $\eta$ values), should occur without regard to the specific task that results from intentional selection. That is, whereas the bottom-up effects may vary as a function of factors such as preparation time (Arrington, 2008), working memory load (Demanet et al., 2010), and mind wandering (Demanet, DeBaene, Arrington, \& Brass, 2013), they should not be differentially engaged on the basis of task frequency, though see the comment below about tasks of asymmetric strengths. Thus, if the less frequent task is the one less likely to be established prior to stimulus onset, the impact of stimulus- 
based factors that draw participants to select this task will be relatively greater.

This account of the present findings relies only on the differential frequencies with which the top-down goal to perform each tasks occurs, not on the differential strengths of the top-down goals for the two tasks. Studies using asymmetric tasks (i.e., Stoop color naming and word reading) have shown that task choice is biased when tasks differ in strength (Liefooghe et al., 2010; Poljac \& Yeung, 2014; Yeung, 2010). Accounts of these findings suggest that the top-down goal to perform the harder task (i.e., color naming) must be more strongly maintained, thus making it more difficult to switch to the easier task (i.e., word reading), and ironically resulting in biases toward performing the harder task. The effect of differential strengths between tasks may change the rate of bottom-up intrusions for each task, with more strongly instantiated tasks being less likely to be interrupted by stimulus priming. There is some suggestion in the asymmetric switch costs in the RT data for the $75 / 25$ condition in Experiment 1 (see the Supplemental materials) that, with extreme differences in frequency, two tasks may start to vary in task strength; however, the present findings are unlikely to be driven by differences in task strength, given that they appear across conditions that do not show asymmetric switch costs. Further research with tasks specifically designed to manipulate task strength will be needed to assess the impact of task strength on the stimulus availability effect.

Contrasting the two experiments provides further insight into the influence of environmental stimuli on task selection. Experiment 1 featured a constrained environment in which the instructions dictated both task frequency and transitions, whereas Experiment 2 was less constrained, with no instructions guiding task selection. Looking across the experiments, the influence of stimulus availability was greater in Experiment 2, when fewer constraints guided task selection, suggesting that as top-down goals are minimized, participants may rely more on environmental influences to guide task selection (Arrington et al., 2014). Furthermore, these experiments make an important methodological point about the VTS paradigm. A question frequently arises: Can anything learned from a paradigm so constrained as to require participants to perform tasks in a random order be applied to cognitive control processes in "real" multitask environments? The similar effects of task frequency on stimulus-based selection processes across the two experiments suggest that the "constrained" environment in Experiment 1 and the "unconstrained" environment in Experiment 2 likely engaged the same underlying mechanisms balancing the influences of top-down and bottom-up control over task selection. As such, researchers interested in studying the cognitive control mechanisms involved in multitasking may prefer the tighter experimental control afforded by more standard instructions in VTS, and may additionally want to avoid the costs associated with the unconstrained versions (i.e., running 66 participants to obtain 16 that provide useable data).

In sum, task choice in the VTS paradigm shows clear influences of both top-down and bottom-up factors in task selection. When considering tasks of unequal frequencies, less frequent tasks show a larger influence of the external environment. In real-world multitask environments, in which some tasks occur relatively infrequently, it may be particularly important to consider environmental cues that drive task selection.

Author note This research was supported by the National Institutes of Health under Grant No. R03 MH082216-01A2 to the first author.

\section{References}

Arrington, C. M. (2008). The effect of stimulus availability on task choice in voluntary task switching. Memory \& Cognition, 38, 991-997. doi:10.3758/MC.36.5.991

Arrington, C. M., \& Logan, G. D. (2004). The cost of a voluntary task switch. Psychological Science, 15, 610-615. doi:10.1111/j.09567976.2004.00728.x

Arrington, C. M., \& Logan, G. D. (2005). Voluntary task switching: Chasing the elusive homunculus. Journal of Experimental Psychology: Learning, Memory, and Cognition, 31, 683-702. doi: 10.1037/0278-7393.31.4.683

Arrington, C. M., Reiman, K. M., \& Weaver, S. M. (2014). Voluntary task switching. In J. Grange \& G. Houghton (Eds.), Task switching (pp. 117-136). Oxford, UK: Oxford University Press.

Arrington, C. M., \& Rhodes, K. M. (2010). Perceptual asymmetries influence task choice: The effect of lateralised presentation of hierarchical stimuli. Laterality, 15, 501-513. doi:10.1080/ 13576500902984695

Arrington, C. M., \& Weaver, S. M. (in press). Rethinking volitional control over task choice in multitask environments: Use of a stimulus set selection strategy in voluntary task switching. Quarterly Journal of Experimental Psychology. doi:10.1080/17470218.2014. 961935

Arrington, C. M., Weaver, S. M., \& Pauker, R. L. (2010). Stimulus-based priming of task choice during voluntary task switching. Journal of Experimental Psychology: Learning, Memory, and Cognition, 36, 1060-1067. doi:10.1037/a0019646

Butler, K. M., Arrington, C. M., \& Weywadt, C. (2011). Working memory capacity modulates task performance but has little influence on task choice. Memory \& Cognition, 39, 708-724. doi:10.3758/ s13421-010-0055-y

Demanet, J., DeBaene, W., Arrington, C. M., \& Brass, M. (2013). Biasing free choices: The role of the rostral cingulate zone in intentional control. NeuroImage, 72, 207-213. doi:10.1016/j.neuroimage.2013. 01.052

Demanet, J., \& Liefooghe, B. (2014). Component processes in voluntary task switching. Quarterly Journal of Experimental Psychology, 67, 843-860. doi:10.1080/17470218.2013.836232

Demanet, J., Verbruggen, F., Liefooghe, B., \& Vandierendonck, A. (2010). Voluntary task switching under load: Contribution of topdown and bottom-up factors in goal-directed behavior. Psychonomic Bulletin \& Review, 3, 387-393. doi:10.3758/PBR.17.3.387

Dreisbach, G., \& Haider, H. (2006). Preparatory adjustment of cognitive control in the task switching paradigm. Psychonomic Bulletin \& Review, 13, 334-338. doi:10.3758/BF03193853 
Haggard, P. (2008). Human volition: Towards a neuroscience of will. Nature Reviews Neuroscience, 9, 934-946. doi:10.1038/nrn2497

Kessler, Y., Shencar, Y., \& Meiran, N. (2009). Choosing to switch: Spontaneous task switching despite associated behavioral costs. Acta Psychologica, 131, 120-128. doi:10.1016/j.actpsy.2009.03. 005

Krieghoff, V., Waszak, F., Prinz, W., \& Brass, M. (2011). Neural and behavioral correlates of intentional actions. Neuropsychologia, 49, 767-776. doi:10.1016/j.neuropsychologia.2011.01.025

Liefooghe, B., Demanet, J., \& Vandierendonck, A. (2010). Persisting activation in voluntary task switching: It all depends on the instructions. Psychonomic Bulletin \& Review, 17, 381-386. doi:10.3758/ PBR.17.3.381

Logan, G. D., \& Gordon, R. D. (2001). Executive control of visual attention in dual-task situations. Psychological Review, 108, 393434. doi:10.1037/0033-295x.108.2.393

Mayr, U., \& Bell, T. (2006). On how to be unpredictable: Evidence from the voluntary task switching paradigm. Psychological Science, 17, 774-780. doi:10.1111/j.1467-9280.2006.01781.x

Monsell, S., Yeung, N., \& Azuma, R. (2000). Reconfiguration of task-set: Is it easier to switch to the weaker task? Psychological Research, 63, 250-264. doi:10.1007/s004269900005

Orr, J. M., Carp, J., \& Weissman, D. H. (2012). The influence of response conflict on voluntary task switching: A novel test of the conflict monitoring model. Psychological Research, 76, 60-73. doi:10. 1007/s00426-011-0324-9
Orr, J. M., \& Weissman, D. H. (2011). Succumbing to bottom-up biases on task choice predicts increased switch costs in the voluntary task switching paradigm. Frontiers in Psychology, 2, 31. doi:10.3389/ fpsyg.2011.00031

Poljac, E., \& Yeung, N. (2014). Dissociable neural correlates of intention and action preparation in voluntary task switching. Cerebral Cortex, 24, 465-478. doi:10.1093/cercor/bhs326

Schneider, D. W., \& Logan, G. D. (2006). Priming cue encoding by manipulating transition frequency in explicitly cued task switching. Psychonomic Bulletin \& Review, 13, 145-151. doi:10.3758/BF03193826

Soon, C. S., He, A. H., Bode, S., \& Haynes, J.-D. (2013). Predicting free choices for abstract intentions. Proceedings of the National Academy of Sciences, 110, 6217-6222. doi: 10.1073/pnas. 1212218110

Vandierendonck, A., Demanet, J., Liefooghe, B., \& Verbruggen, F. (2012). A chain-retrieval model for voluntary task switching. Cognitive Psychology, 65, 241-283. doi:10.1016/j.cogpsych. 2012.04.003

Yeung, N. (2010). Bottom-up influences on voluntary task switching: The elusive homunculus escapes. Journal of Experimental Psychology: Learning, Memory, and Cognition, 36, 348-362. doi:10.1037/a0017894

Yeung, N., \& Monsell, S. (2003). The effects of recent practice on task switching. Journal of Experimental Psychology: Human Perception and Performance, 29, 919-936. doi:10.1037/0096-1523.29.5.919 